\title{
ON THE $D$-SERIES OF A FINITE GROUP
}

\section{J. BUCKLEY}

Introduction. Let $G$ be a group. The lower central series of $G$ is defined inductively by: $G_{1}=G$ and $G_{k+1}=\left[G_{k}, G\right]$ the subgroup of $G$ generated by the set of all commutators $[\sigma, \tau]$ where $\sigma \in G_{k}$ and $\tau \in G$. By definition $G$ is nilpotent if $G_{k}=1$ for some positive integer $k$.

Let $Z[G]$ denote the integral group ring of $G$ and $I$ the ideal spanned by the elements $\sigma-1$ where $\sigma \in G$. $I$ is called the augmentation ideal or the fundamental ideal of $Z[G]$ and the powers of $I$ give rise to the descending series of fully invariant subgroups of $G$,

$$
\cdots \subset D_{2}(G) \subset D_{1}(G)=G
$$

where $D_{k}(G)$ consists of those elements $\sigma$ in $G$ such that $\sigma \equiv 1\left(\bmod I^{k}\right)$. We refer to this as the $D$-series of $G$.

For each $k, G_{k} \subset D_{k}(G)$ and the map $\Delta: \sigma \rightarrow \sigma-1$ induces a homomorphism $\Delta_{k}$ from $G_{k} / G_{k+1}$ into $I^{k} / I^{k+1}$. The image of $\Delta_{k}$ is the set of homogeneous Lie elements (in the $\Delta \sigma$ ) in the module $I^{k} / I^{k+1}$, and the kernel is $G_{k} \cap D_{k+1} / G_{k+1}$. (See Cohn [1].) Also $G_{2}=D_{2}(G)$ for all groups, and all $G_{k}=D_{k}(G)$ if $G$ is a free group. (This last result is due to Magnus [5].)

It has been conjectured that all $G_{k}=D_{k}(G)$ for arbitrary groups $G$. We do not prove this, but we do show that for finite groups $\cap G_{k}$ $=\cap D_{k}(G)$, or, in other words, that the groups at which the two descending series become constant coincide. This has the immediate corollary that a finite group $G$ is nilpotent if and only if $D_{k}(G)=1$ for some positive in teger $k$.

We begin with the following characterization of $p$-groups among all finite groups. This theorem appears in a paper of Gruenberg [1]. We give a simple proof of it here.

THEOREM 1. Let $G$ be a finite group. $G$ is a p-group for some prime $p$ if and only if $\cap I^{k}=0$.

Proof. Suppose $G$ is a finite $p$-group for some prime $p$. The canonical ring homomorphism from $Z[G]$ to $\Gamma[G]$, the group algebra of $G$ over $G F(p)$ the field with $p$ elements, takes $I$ onto the augmentation ideal $\Delta$ of $\Gamma[G]$. It is known (Jennings $[4]$ ) that $\Delta$ is precisely the radical of $\Gamma[G]$ and hence $\Delta^{l}=0$ for some positive integer $l$. Consequently $I^{l} \subset p Z[G]$. Hence $\cap I^{k} \subset \cap p^{k} Z[G]$ which is clearly 0 .

Conversely, if $\cap I^{k}=0$, it follows that $\cap D_{k}(G)=1$ and hence that $\cap G_{k}=1$. Since $G$ is finite, this implies that $G$ is nilpotent, hence a direct product of $p$-groups. To show $G$ is a $p$-group, we show that if $G$

Received by the editors June 10, 1966. 
contains commuting elements $\sigma$ and $\tau$ of different prime power orders $p$ and $q$, then $\cap I^{k} \neq 0$. If $\sigma^{\prime}=\sigma^{p-1}+\cdots+1$ and $\tau^{\prime}=\tau^{q-1}+\cdots+1$, then $(\sigma-1) \sigma^{\prime}=0$ and $(\tau-1) \tau^{\prime}=0$. Let $a p+b q=1$, and set $\alpha=a \sigma^{\prime}$ $+b \tau^{\prime} ;$ then $(\sigma-1)(\tau-1) \alpha=0$. Under the augmentation map, $\alpha$ goes in to $a p+b q=1$, whence $\alpha=1-\beta$ for some $\beta$ in $I$. Therefore $\beta(\sigma-1)(\tau-1)=(\sigma-1)(\tau-1)$, and since $\beta \in I$ and $(\sigma-1)(\tau-1) \in I^{2}$, it follows that $(\sigma-1)(\tau-1) \in I^{k}$ for all $k$.

The following result proves useful. We omit the proof which is straightforward.

Lemma 1. Let $G$ be a finite group. Then $\cap G_{k}=\cap N$ where $N$ runs through all normal subgroups of $G$ of prime power index.

Let $N$ be a normal subgroup of $G$. The following standard facts can be found in Fox [2]. The kernel of the canonical ring homomorphism $Z[G] \rightarrow Z[G / N]$ is the ideal generated by the set of all $\tau-1, \tau \in N$. If we denote this ideal by $I(N)$, then $Z[G / N] \simeq Z[G] / I(N)$. Also, if $\sigma \in G$ and $\sigma-1 \in I(N)$, then $\sigma \in N$.

Lemma 2. Let $N$ be a normal subgroup of $G$. $N$ has prime power index in $G$ if and only if $I(N)=\bigcap\left[I(N)+I^{k}\right]$.

Proof. If we identify $Z[G / N]$ with $Z[G] / I(N)$, the $k$ th power of the augmentation ideal of $Z[G / N]$ is identified with $\left[I(N)+I^{k}\right] / I(N)$. The result now follows immediately from Theorem 1.

Theorem 2. Let $G$ be a finite group. Then $\cap G_{k}=\cap D_{k}(G)$.

Proof. Since $G_{k} \subset D_{k}(G)$ for all $k$, the inclusion one way is clear. To show $\cap D_{k}(G) \subset \cap G_{k}$, let $\sigma \in \cap D_{k}(G)$. Then $\sigma-1 \in \cap I^{k}$, whence for all normal $N$ of prime power index, $\sigma-1 \in I(N)$. Therefore $\sigma \in N$ for all such $N$, and $\sigma \in \cap G_{k}$.

Corollary 1. Let $G$ be a finite group. $G$ is nilpotent if and only if $D_{k}(G)=1$ for some positive integer $k$.

\section{REFERENCES}

1. P. M. Cohn, Generalization of a theorem of Magnus, Proc. London Math. Soc. 57 (1952), 297-310. (See correction in same volume.)

2. R. H. Fox, Free differential calculus. I, Ann. of Math. 57 (1953), 547-560.

3. K. Gruenberg, The residual nilpotence of certain presentations of finite groups, Arch. Math. 13 (1962), 410-417.

4. S. A. Jennings, The structure of the group ring of a p-group over a modular field, Trans. Amer. Math. Soc. 50 (1941), 175-185.

5. W. Magnus, Über Beziehungen zwischen höheren Kommutatoren, J. Reine Angew. Math. 177 (1937), 105-115.

Dartmouth College 